\title{
Spacetimes foliated by non-expanding null surfaces in the presence of a cosmological constant
}

\author{
Jerzy Lewandowski and Adam Szereszewski \\ Faculty of Physics, University of Warsaw, Pasteura 5, 02-093 Warsaw, Poland
}

(Dated: September 21, 2018)

\begin{abstract}
We prove that every solution to Einstein's equations with possibly non-zero cosmological constant that is foliated by non-expanding null surfaces transversal to a single non-expanding null surface belongs to family of the near (extremal) horizon geometries. Our results are local, hold in a neighborhood of the single non-expanding null surface.
\end{abstract}

PACS numbers:

\section{INTRODUCTION}

Spacetimes foliated by non-expanding null surfaces were introduced by Kundt in the context of explicit ("exact") solutions to Einstein's equations describing plane fronted gravitational waves [1]. In that framework the primary structure is a congruence of null geodesic curves that is non-diverging and surface forming 2]. Another topic of General Relativity in which non-expanding null surfaces appear is theory of non-expanding horizons (NEHs) [3, 4]. Within that theory a geometric description of NEHs was developed, the constraints following from the Einstein's equations satisfied by a surrounding $n+2$ dimensional spacetime $M$ were studied. Upon some energy assumptions that are satisfied by all the solutions of the vacuum Einstein's equations with a possibly non-zero cosmological constant considered in the current paper, the internal geometric structure induced on every NEH $H$ was identified as a degenerate metric tensor $g_{a b}$ of the signature $(0+\ldots+)$ and a torsion free covariant derivative $\nabla_{a}$ such that

$$
\nabla_{a} g_{b c}=0 .
$$

While every null vector field $\ell$ tangent to $H$ satisfies

$$
\mathcal{L}_{\ell} g_{a b}=0
$$

identically, the geometry of $H$ may admit a stronger symmetry generator $\ell$ that satisfies

$$
\left[\mathcal{L}_{\ell}, \nabla_{a}\right]=0 .
$$

In the latter case $H$ is called an isolated horizon. A rotation 1-form potential $\omega^{(\ell)}$ of a NEH $H$, depends on choice of a null vector $\ell$, and is defined by

$$
\nabla_{a} \ell=\omega_{a}^{(\ell)} \ell
$$

It satisfies the zeroth law of NEH mechanics

$$
\nabla_{a} \kappa^{(\ell)}=\mathcal{L}_{\ell} \omega^{(\ell)}, \quad \kappa^{(\ell)}:=\ell^{a} \omega_{a}^{(\ell)} .
$$

For every isolated horizon

$$
\kappa^{(\ell)}=\text { const }
$$

and the horizon is called extremal whenever

$$
\kappa^{(\ell)}=0
$$

A geometrical identity that connects the internal and external geometry of an extremal isolated horizon $(\tilde{H}, \tilde{\ell})$ turns into a constraint on data induced on an $n$-dimensional section $S$ of $\tilde{H}$ : the metric tensor $g_{A B}$, its torsion free metric connection $\nabla_{A}^{(n)}$ and Ricci tensor $R_{A B}^{(n)}$, and the pullback of the rotation 1-form $\omega_{A}^{(\tilde{\ell})}$ on the one hand, and the spacetime Ricci tensor $R_{\alpha \beta}$ on the other hand, namely [3, 4]

$$
-\nabla_{(A}^{(n)} \omega_{B)}^{(\tilde{\ell})}-\omega_{A}^{(\tilde{\ell})} \omega_{B}^{(\tilde{\ell})}+\frac{1}{2} R_{A B}^{(n)}=\frac{1}{2} R_{A B} .
$$


That geometric point of view can also be applied to non-expanding null surfaces

$$
\mathbb{R} \ni u \mapsto H_{u} \subset M
$$

foliating a Kund's class spacetime $M$. The existence of a foliation may be used to distinguish a family of null vector fields in spacetime by

$$
\ell_{\mu}=-\nabla_{\mu} u
$$

For every lief $H_{u}$, the corresponding vector field $\ell^{\mu}$ is tangent, and satisfies

$$
\kappa^{(\ell)}=0
$$

Since the function $u$ is defined up to transformations

$$
u^{\prime}=f(u)
$$

it follows that $\ell$ is defined up to a factor $f^{\prime}(u)$ constant on every $\mathrm{NEH} H_{u}$. The most intriguing is a constraint implied by the existence of the foliation, and constraining the data induced in every $n$-dimensional section of $H_{u}$, namely [4]

$$
\nabla_{(A}^{(n)} \omega_{B)}^{(\ell)}-\omega_{A}^{(\ell)} \omega_{B}^{(\ell)}+\frac{1}{2} R_{A B}^{(n)}=\frac{1}{2} R_{A B}
$$

The first, easy observation is, that given a solution $g_{A B}, \omega_{A}^{(\ell)}$ of the foliation constraint (13) we can transform it into a solution $g_{A B}, \omega_{A}^{(\tilde{\ell})}$,

$$
\omega_{A}^{(\tilde{\ell})}:=-\omega_{A}^{(\ell)}
$$

of the extremal isolated horizon equation (8). Secondly, it turns out, that if $H$ is just any NEH (no foliation assumption), transversal to an extremal isolated horizon $(\tilde{H}, \tilde{\ell})$, and a null vector field $\ell$ tangent to $H$ is normalized at the intersection $S$, such that

$$
\tilde{\ell}^{\mu} \ell_{\mu}=\mathrm{const}
$$

then the geometry of $H$ satisfies the foliation constraint (13) at $S$ (even if no foliation exists) [5, 6]. Hence, the foliation constraint may be induced by a transversal isolated horizon.

The second observation led to the construction of a family of solutions to vacuum Einstein's equations in 4dimensional spacetime foliated by bifurcated Killing horizons whose common part is an extremal Killing horizon [5, 6]. Every solution in that family is determined by a metric tensor $g_{A B}$ and a 1-form $\omega_{A}$ defined on a 2 -dimensional manifold $S$ and satisfying the following equation

$$
\nabla_{(A}^{(2)} \omega_{B)}-\omega_{A} \omega_{B}+\frac{1}{2} R_{A B}^{(2)}=0
$$

The corresponding metric tensor is defined on $M=S \times \mathbb{R} \times \mathbb{R}$ in coordinates $\left(x^{\mu}\right)=\left(x^{A}, v, u\right)$ as follows

$$
g_{\mu \nu} d x^{\mu} d x^{\nu}=g_{A B} d x^{A} d x^{B}-2 d u\left(d v+2 v \omega+\frac{1}{2} v^{2}\left(\nabla_{A} \omega^{A}-2 \omega_{A} \omega^{A}\right) d u\right) .
$$

The foliation consists of surfaces

$$
H_{u}: u=u_{0}, \quad u_{0} \in \mathbb{R}
$$

the transversal extremal Killing horizon is the surface

$$
\tilde{H}: v=0
$$

the extremal Killing vector is $\partial_{u}$ while the non-extremal Killing vectors are $v \partial_{v}-\left(u-u_{0}\right) \partial_{u}$. A spacetime (17) is a generalization of the solutions obtained by the Bardeen-Horowitz limit from extremal Kerr black hole solutions and these days is known better as near horizon geometry [7 9]. They were generalized to higher dimensions, to $\Lambda \neq 0$, and to various kinds of matter fields [6, 9]. 


\section{KUND'S CLASS SPACETIMES CONTAINING A TRANSVERSAL NEH}

The mechanism of inducing the foliation constraint (13) by existence of a transversal isolated horizon suggest a natural characterization of Kund's class metric by existence or not of a transversal extremal isolated horizon. In fact, a transversal NEH will automatically admit a null symmetry that will make it isolated horizon [6]. We follow that clue and divide the Kunds class spacetimes into two types:

(i) containing a transversal NEH (possibly, after the extension along the null generators of the lives of the foliation)

(ii) not containing any transversal NEH

In the current paper we consider Kund's class spacetimes of the type (i) above. We find all the solutions to the vacuum Einstein equations with possibly non-zero cosmological constant in 4-dimensional spacetime,

$$
R_{\mu \nu}=\Lambda g_{\mu \nu}
$$

Our derivation applies also to arbitrary dimension $n \geq 4$ spacetime if some generalization of the characteristic Cauchy problem is true there.

\section{A. Example: NHG spacetime}

An example of a spacetimes we are looking for is the metric tensor defined on

$$
M=S \times \mathbb{R} \times \mathbb{R}
$$

in coordinates system $\left(x^{\mu}\right)=\left(x^{A}, v, u\right)$ corresponding to the product as follows

$$
g_{\mu \nu} d x^{\mu} d x^{\nu}=g_{A B}(x) d x^{A} d x^{B}-2 d u\left(d v+2 v \omega_{A}(x) d x^{A}+\frac{1}{2} v^{2}\left(\nabla_{A}(x) \omega^{A}(x)-2 \omega_{A}(x) \omega^{A}(x)-\Lambda\right) d u\right)
$$

where the 1-form $\omega_{A}(x)$ and metric tensor $g_{A B}(x)$ defined on $S$ satisfy the foliation constraint

$$
\nabla_{(A}^{(2)} \omega_{B)}-\omega_{A} \omega_{B}+\frac{1}{2} R_{A B}^{(2)}-\frac{1}{2} \Lambda g_{A B}=0
$$

The NEH foliation is given by

$$
H_{u}=S \times \mathbb{R} \times\{u\}, \quad u \in \mathbb{R} .
$$

The transversal NEH is

$$
\tilde{H}=S \times\{0\} \times \mathbb{R}
$$

The vector field

$$
\ell:=\partial_{v}
$$

satisfies

$$
\ell_{\mu}=-\nabla_{\mu} u
$$

as required. For every $u$, on the $\mathrm{NEH} S \times \mathbb{R} \times\{u\}$, the rotation 1 -form potential is

$$
\omega^{(\ell)}=\omega_{A}(x) d x^{A} .
$$

By inspection one can check that (17, 20) satisfies the Einstein's equations (18). 


\section{B. Einstein spacetimes foliated by NEHs transversal to a single NEH}

Consider a general spacetime of the type (i) above, that is foliated by non-expanding null surfaces transversal to a single non-expanding null surface and assume it satisfies the Einstein's equations

$$
R_{\mu \nu}=\Lambda g_{\mu \nu}
$$

Let

$$
\mathbb{R} \ni u \mapsto H_{u}
$$

be the family of non-expanding null surfaces, and $\tilde{H}$ be the transversal non-expanding null surface. Locally, in a suitable neighborhood of a point of $\tilde{H}$, we choose the function $u$ to be one of spacetime coordinates. A vector field $\ell^{\mu}$ defined by

$$
\ell_{\mu}=-\nabla_{\mu} u
$$

is tangent to each of the leaves $H_{u}$, null, and satisfies

$$
\ell^{\mu} \nabla_{\mu} \ell=0
$$

Define the second coordinate, a function $v$, such that

$$
\ell^{\mu} \nabla_{\mu} v=1, \quad \text { and } \quad v_{\mid \tilde{H}}=0
$$

Locally, for every point of $\tilde{H}$, we may consider a neighborhood in $M$, such that every $H_{u}$ and $\tilde{H}$ have the topology

$$
H_{u}, \tilde{H}=S \times \mathbb{R}
$$

and the intersection is

$$
H_{u} \cap \tilde{H}=S,
$$

where $S$ is a 2-dimensional manifold. The remaining two spacetime coordinates $x^{A}, A=1,2$ are introduced first on $S$, next pass to $H_{0} \cap \tilde{H}$, next extended along $\tilde{H}$ such that they are constant along the null generators, and finally along each $H_{u}$ by

$$
\ell^{\mu} \nabla_{\mu} x^{A}=0
$$

In the resulting coordinates

$$
\left(x^{\mu}\right)=\left(x^{A}, v, u\right)
$$

the metric tensor $g$ takes the following form

$$
g_{\mu \nu}(x, v, u) d x^{\mu} d x^{\nu}=g_{A B}(x, v, u) d x^{A} d x^{B}-2 d u\left(d v+W_{A}(x, v, u) d x^{A}+H(x, v, u) d u\right),
$$

where the dependence on the coordinates $v$ and $u$ is restricted by the properties of non-expanding null surfaces. Indeed, to begin with

$$
g_{A B}(x, v, u)_{, v}=0, \quad \text { and } \quad g_{A B}(x, 0, u)_{, u}=0 .
$$

Due to the Einstein's equations (18) the first equality follows from the non-expanding of each $H_{u}$, and the second equality follows from the non-expanding of $\tilde{H}$. Consequently,

$$
g_{A B}(x, v, u)=g_{A B}(x)
$$

is a unique metric tensor on $S$.

Secondly, the second assumption in (30) about $v$ implies

$$
W_{A}(x, 0, u)=0=H(x, 0, u) .
$$


Next, for every $H_{u}$, the rotation 1 -form potential is

$$
\omega_{A}^{(\ell)}=\frac{1}{2} W_{A, v}
$$

The 0th law (5) and (29) imply

$$
W_{A, v v}(x, v, u)=0 .
$$

Hence,

$$
W_{A}(x, v, u)=2 v \omega_{A}(x, u) .
$$

One can also notice, that at the transversal non-expanding null surface $\tilde{H}$, as a tangent null vector field we can use

$$
\tilde{\ell}:=\partial_{u}
$$

Since by the construction

$$
\tilde{\ell}^{\mu} \ell_{\mu}=-\partial_{u} u=-1
$$

the 1 -form $\omega_{A}$ is also related to the rotation 1 -form potential on $\tilde{H}$,

$$
\omega^{(\tilde{\ell})}(x, u)=\kappa^{(\tilde{\ell})}(x) d u-\omega_{A}(x, u) d x^{A} .
$$

Of course the 0th law (5) still applies, but at this point we do not know anything else about the dependence of $u$ and $x$.

In order to show that $\omega_{A}(x, u)$ is independent of $u$, we will refer to the holographic characterization of a spacetime that contains two transversal NEHs [10]. Consider a 4-dimensional spacetime $M$, equipped with a metric tensor $g_{\mu \nu}$ that satisfies the vacuum Einstein's equations with given cosmological constant $\Lambda$. Suppose $H \subset M$ and $\tilde{H} \subset M$ are two NEHs, and

$$
S=H \cap \tilde{H}
$$

is a 2-dimensional spacelike surface in $M$. Then, the metric tensor $g_{\mu \nu}$ in the future and in the past of $S$ is locally, near $S$, and up to diffeomorphisms, determined by the following holographic data induced on $S$ :

- the induced metric tensor $g_{A B}$,

- the pullback $\omega_{A B}$ of the rotation 1-form potential $\omega_{a}^{(\ell)}$ defined on $H$ by a tangent, null vector field $\ell$.

Moreover, two data sets, $\left(g_{1}, \omega_{1}\right)$ and $\left(g_{2}, \omega_{2}\right)$ related either by a diffeomorphism of $S$ or by

$$
\omega_{2}=\omega_{1}+d f, \quad g_{1}=g_{2}
$$

define diffeomorphically equivalent metric tensors.

We go back now to the metric tensor (35). Choose any value $u_{0}$ of the coordinate $u$ and consider the holographic data $g_{A B}(x)$ and $\omega_{A}^{(\ell)}\left(x, u_{0}\right)$ defined on the intersection

$$
S_{u_{0}}=H_{u_{0}} \cap \tilde{H}=S \times\{0\} \times\left\{u_{0}\right\}
$$

Next we notice, that among the spacetimes (19) there is one, that defines the same (up to diffeomorphisms) holographic data. To construct it, just set in the definition of the metric tensor (19) the following

$$
g_{A B}(x):=g_{A B}(x), \quad \omega_{A}\left(x, u_{0}\right):=\omega_{A}^{(\ell)}\left(x, 0, u_{0}\right),
$$

defined on $S$, considering $u_{0}$ in $\omega_{A}$ as a parameter. Denote the resulting metric by $g_{\left(u_{0}\right)}$. The holographic data it defines on

$$
S_{u_{0}^{\prime}}=H_{u_{0}^{\prime}} \cap \tilde{H}=S \times\{0\} \times\left\{u_{0}^{\prime}\right\}
$$

for arbitrarily fixed $u_{0}^{\prime}$ is the same (up to the diffeomorphism) as the holographic data defined on $S_{u_{0}}$ by the metric $g$. The conclusion is, that the metric tensor $g$ (35) restricted to the future and past of $S_{u_{0}}$ is isometric to the metric 
$g_{\left(u_{0}\right)}$ (19) restricted to the future and the past of $S_{u_{0}^{\prime}}$. What may happen is, that the coordinates $\left(x^{A}, v, u\right)$ are not preserved by that isometry. However they transform within the remaining freedom,

$$
u^{\prime}=U(u), \quad v^{\prime}=\frac{v}{U^{\prime}(u)}, \quad x^{\prime A}=x^{A} .
$$

Therefore without lack of generality, we change them in (35) such that actually the isometry preserves the coordinates. We can also fix $u_{0}^{\prime}=0$. In those coordinates the components $W_{A}(x, v, u)$ and $H(x, v, u)$ in (35) coincide with the corresponding components of the metric (19) for $u, v>0$ and for $u, v<0$. In the case of $W_{A}$ it means that

$$
2 v \omega_{A}(x, u)=2 v \omega_{A}(x), \quad \text { for } \quad u, v>0, u, v<0 .
$$

This is sufficient to conclude that

$$
\omega_{A}(x, u)=\omega_{A}(x)
$$

meaning that in (35),

$$
W_{A}(x, v, u)=2 v \omega_{A}(x) .
$$

To determine the function $H(x, v, u)$ in (35), we just conclude from the comparison with (19) in the domain $v, u<0$ or $v, u>0$, by the second differentiability of $H$, that

$$
H(x, 0, u)_{, v}=0 .
$$

With the initial conditions (38) (the second equation) and (49) at $v=0$, we can integrate the Einstein's equation given by the component $R_{u v}$ of the Ricci tensor. We calculate $R_{u v}$ for the metric (35) with (48) (the first equation below) and use the Einstein's equations (the second equation),

$$
R_{u v}=-\nabla_{A}^{(n)} \omega^{A}+2 \omega^{A} \omega_{A}+H_{, v v}=-\Lambda .
$$

The result is

$$
H(x, v)=\frac{1}{2}\left(\nabla_{A} \omega^{A}-2 \omega_{A} \omega^{A}-\Lambda\right) v^{2} .
$$

and as it could be anticipated, it coincides with (19).

\section{SUMMARY}

In summary, we have proved the following

Theorem: Suppose $M$ is a 4-dimensional spacetime equipped with a metric tensor $g$ that satisfies Einstein's equations

$$
R_{\mu \nu}=\Lambda g_{\mu \nu},
$$

and admits a foliation by non-expanding null surfaces transversal to a single non-expanding null surface $\tilde{H}$. Locally, in a neighborhood of every point of $\tilde{H}$, the metric $g$ can be written in the form (19, 20).

The resulting spacetime is the known near horizon geometry. It has 2-dimensional family of Killing vectors, linear combinations of:

$$
K:=\partial_{u}, \quad L:=u \partial_{u}-v \partial_{v} .
$$

The non-expanding surfaces $u=\mathrm{u}_{0}$ and $v=0$ are Killing Horizons of $\left(u-u_{0}\right) \partial_{u}-v \partial_{v}$ while $v=0$ is at the same time the extremal Killing horizon of $K$ [11, 12].

Our result generalizes easily to an arbitrary dimension $n$ provided it continues to be true that a solution to the vacuum Einstein's equations with cosmological constant that admits two intersecting generically non-expanding null surfaces $H$ and $H^{\prime}$ is uniquely determined (locally, in a neighborhood of the intersection) in the future and past of the intersection by internal geometries $g_{a b}, \nabla_{a}$ and $g_{a b}^{\prime}, \nabla_{a}^{\prime}$ induced on $H$, and, respectively $H^{\prime}$.

Acknowledgments. This work was partially supported by the Polish National Science Centre grant No. 2015/17/B/ST2/02871 .

[1] W. Kundt, The plane-fronted gravitational waves, Z. Physik 163, 77-86 (1961). 
[2] J. Podolský and M. Ortaggio, Explicit Kundt type $I I$ and $N$ solutions as gravitational waves in various type $D$ and $O$ universes, Class. Quantum Grav. 20, 1685-1701 (2003).

[3] A. Ashtekar, C. Beetle, and J. Lewandowski, Geometry of Generic Isolated Horizon, Class. Quantum Grav. 19, 1195-1225 (2002), arXiv:gr-qc/0111067.

[4] J. Lewandowski and T. Pawłowski, Quasi-local rotating black holes in higher dimension: Geometry, Classical Quantum Gravity 22, 1573 (2005).

[5] T. Pawłowski, J. Lewandowski, and J. Jezierski, Spacetimes foliated by Killing horizons, Class. Quantum Grav. 21, 12371252 (2004), arXiv:gr-qc/0306107.

[6] J. Lewandowski, A. Szereszewski, and P. Waluk, Spacetimes foliated by non-expanding and Killing horizons: higher dimension, Phys. Rev. D 94, 064018 (2016).

[7] J.M. Bardeen and G.T. Horowitz, Extreme Kerr throat geometry: A Vacuum analog of $A d S(2) \times S^{2}$, Phys. Rev. D 60, 104030 (1999), arXiv:hep-th/9905099.

[8] H. S. Reall, Higher dimensional black holes and supersymmetry, Phys. Rev. D 68, 024024 (2003).

[9] H.K. Kunduri and J. Lucietti, Classification of Near-Horizon Geometries of Extremal Black Holes, Living Rev. Rel. 16 (2013), 8, http://www.livingreviews.org/lrr-2013-8, arXiv:abs/1306.2517.

[10] I. Rácz, Stationary black holes as holographs II., Class. Quantum Grav. 31, 035006 (2014).

[11] Mars, M., Paetz, T.-T, and Senovilla, J.M.M., Multiple Killing Horizons, Class. Quantum Grav. 35 (2018) 155015; arXiv:1803:03054

[12] M. Mars, T.-T Paetz, and J.M.M. Senovilla, Multiple Killing Horizons and Near Horizon Geometries, arXiv:1807.02679 